\title{
Walking and running on yielding and fluidizing ground
}

\author{
Feifei Qian ${ }^{1}$, Tingnan Zhang ${ }^{1}$, Chen $\mathrm{Li}^{1}$, Pierangelo Masarati ${ }^{4}$, Aaron M. Hoover ${ }^{3}$, Paul Birkmeyer ${ }^{2}$, \\ Andrew Pullin ${ }^{2}$, Ronald S. Fearing ${ }^{2} \&$ Daniel I. Goldman ${ }^{1}$ \\ ${ }^{1}$ Georgia Institute of Technology, Atlanta, Georgia, \\ USA, 30332-0250 \\ ${ }^{2}$ University of California at Berkeley, Berkeley, USA, \\ 94720
}

\begin{abstract}
We study the detailed locomotor mechanics of a small, lightweight robot (DynaRoACH, $10 \mathrm{~cm}, 25 \mathrm{~g}$ ) which can move on a granular substrate of closely packed $3 \mathrm{~mm}$ diameter glass particles at speeds up to $50 \mathrm{~cm} / \mathrm{s}(5 \mathrm{body}$ length/s), approaching the performance of small, highperforming, desert-dwelling lizards. To reveal how the robot achieves this high performance, we used high speed imaging to capture kinematics, and developed a numerical multi-body simulation of the robot coupled to an experimentally validated discrete element method (DEM) simulation of the granular media. Average forward speeds measured in both experiment and simulation agreed well, and increased non-linearly with stride frequency, reflecting a change in the mode of propulsion. At low frequencies, the robot used a quasi-static "rotary walking" mode, in which the granular material yielded as the legs penetrated and then solidified once vertical force balance was achieved. At high frequencies, duty factor decreased below 0.5 and aerial phases occurred. The propulsion mechanism was qualitatively different: the robot ran rapidly by utilizing the speed-dependent fluid-like inertial response of the material. We also used our simulation tool to vary substrate parameters that were inconvenient to vary in experiment (e.g., granular particle friction) to test performance and reveal limits of stability of the robot. Using small robots as physical models, our study reveals a mechanism by which small animals can achieve high performance on granular substrates, which in return advances the design and control of small robots in deformable terrains.
\end{abstract}

Keywords: bio-inspired robot; legged locomotion; granular media; lightweight

\section{INTRODUCTION}

There is an increasing need for robots to traverse a diversity of complex terrain. Platforms have been developed that can effectively run on fractured rigid ground [1], [2], crawl within concave surfaces [3], and climb on walls [4]. However, relative to biological organisms [5], small vehicles often have poor locomotor ability on granular substrates like sand and gravel.
For example, in wheeled and tracked vehicles, wheel slippage and sinkage can cause significant performance loss [6].

Granular media are collections of particles that interact through dissipative, repulsive contact forces [7]. Forced granular media remain solid below the yield stress but flow like a fluid when the yield stress is exceeded [8]. The solid-fluid transition presents great challenges for terrestrial devices moving on granular media. For example, previous studies [9] demonstrated that a bio-inspired RHex-class legged robot, SandBot $(30 \mathrm{~cm}, 2.3 \mathrm{~kg})$, walked effectively at up to 1 body length/s on granular media at low to intermediate stride frequencies, where the granular material behaved like a yielding solid. The granular material yielded as the legs penetrated until vertical force balance was achieved; the granular material then solidified under the legs while the body lifted and moved forward, as if the robot were walking on a solid. At high stride frequencies, however, because the legs encountered previously disturbed ground, the granular material around the legs became continuously fluidized, and the robot "swam" forward slowly ( 0.01 body length/s) using drag on the legs to overcome belly drag.

In contrast, a variety of animals live in the deserts and move rapidly across granular surfaces. For example, the zebra-tailed lizard (Callisaurus draconoides, $\sim 10 \mathrm{~cm}, \sim 10 \mathrm{~g}$ ) can run at speeds of $100 \mathrm{~cm} / \mathrm{s}$ (10 body length/s) on sand. Unlike SandBot which must penetrate a large portion (> 70\%) of its legs to move on granular media, the lizard is light enough that even while running it only penetrates a small portion $(<30 \%)$ of its legs into granular substrates to generate force [5]. This suggests that a small, lightweight body may confer advantages for locomotion on deformable surfaces such as granular media.

Recent advances in smart composite microstructure (SCM) [10] have enabled the development of small, lightweight robots $(\sim 10 \mathrm{~cm}, \sim 20 \mathrm{~g})$ [11] [12] like DynaRoACH (Fig. 1A). These robots are similar in size to the zebra-tailed lizard (among other myriad desert vertebrates and invertebrates [13], [14]) and can achieve performance approaching animals $(\sim 10$ body length/s) on solid surfaces. Therefore, in addition to advancing locomotor capabilities of devices on complex terrain [15], these lightweight robots provide promising physical models to study how effective legged locomotion can be achieved on granular substrates in small, high-performing animals. 
Another challenge for studying locomotion on granular media is the lack of comprehensive force models at the level of the Navier-Stokes equations for fluids [16]. Recently an experimentally validated discrete element method (DEM) simulation (described below) of a model granular medium (3 $\mathrm{mm}$ diameter glass particles) was developed and successfully captured the locomotor mechanics of a sand-swimming lizard moving within granular media [17]. The DEM simulation provides a tool to obtain accurate, detailed information such as force and flow of the granular media during intrusions relevant to locomotion. Such information is challenging to obtain in experiments, since force platforms [18] and 3D particle image velocimetry (PIV) are not yet developed for deformable opaque ground.

In this paper we reveal basic principles of movement of lightweight locomotors on granular media using a combination of laboratory experiment and computer simulation. We perform studies of DynaRoACH on a medium of small glass particles. To obtain estimates of ground reaction forces that result in high performance, we integrate the DEM simulation with a multibody dynamic simulation of the DynaRoACH robot. Our study reveals for the first time that qualitatively different propulsion mechanisms exist for low and high frequency movement on granular media. While the low frequency locomotion of DynaRoACH can be understood using a previously introduced "rotary walking" model, at higher frequency, the robot utilizes a hydrodynamic mechanical response of the granular material to achieve high performance through sustained fluidization of the ground. We use the simulation to systematically vary parameters like particle friction that are inconvenient to modify in experiment, and demonstrate performance and stability limits. We expect that mechanics discovered here and the tools we have developed should be applicable to other devices and provide a starting point to understand biological locomotion and develop robot designs on more complex deformable substrates, like leaf litter and mud.

\section{MATERIALS AND METHODS}

\section{A. Experiments}

\section{1) Robotic platform}

The DynaRoACH robot used in this study (Fig. 1A) is a small, lightweight $(10 \mathrm{~cm}, 25 \mathrm{~g})$, bio-inspired hexapedal robot [11]. It has six c-shaped legs (radius $1 \mathrm{~cm}$ ) and uses an alternating tripod gait. All six legs are driven by a single motor through body linkages. The motor is controlled by a centralized controller mounted close to the center of mass (CoM) of the robot. Control parameters like stride frequency, running time, and PID control gains are set on a PC and communicated to the controller through a Bluetooth wireless interface.

\section{2) Model granular media}

We used $3.0 \pm 0.2 \mathrm{~mm}$ diameter glass particles (density $=$ $2.47 \mathrm{~g} / \mathrm{cm}^{3}$ ) as the granular medium (see Fig. 1C). The large size of the particles reduces computation time in the simulation portion of the study, facilitating a direct comparison between experiment and simulation. While these particles are larger than most natural sand grains, they behave qualitatively similar [8]; a previous study [17] demonstrated that these particles were a good model for studying locomotion within granular media.

In nature, granular media exist in a range of compactions, measured by the volume fraction $\phi$ (the ratio between the solid volume and the occupied volume). For dry granular media $\phi$ can vary from $0.57<\phi<0.64$ [19], although this range is influenced by particle friction [20]. The yield strength of a granular medium generally increases with $\phi$ [9], [21] and affects locomotor performance on the granular medium [9]. In our study, we prepared the granular medium into a closely packed state $(\phi=0.63)$. However, we found that our results did not qualitatively change for different $\phi$ (e.g., robot speed was insensitive to $\phi$ ), likely because the robot penetrates its legs into the granular medium to depths of only a few particle diameters and the range of achievable $\phi$ was small $(0.61<\phi<$ 0.63 ) in the low friction $3 \mathrm{~mm}$ particles.
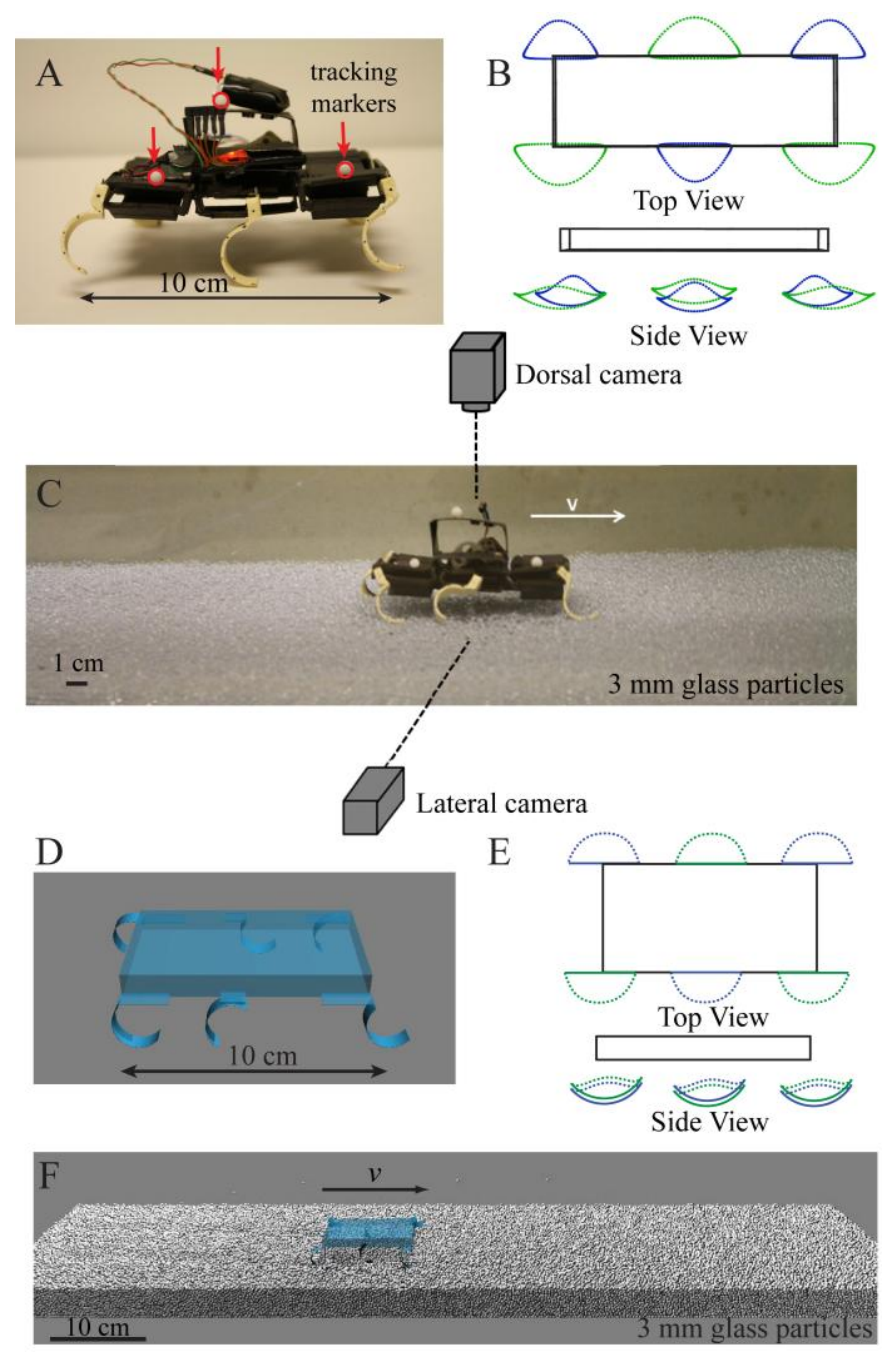

Figure 1. Locomotion experiment and simulation. (A) The lightweight, hexapedal DynaRoACH robot. (B) Leg tip trajectories from top view and side view. Blue and green trajectories denote the two alternating tripods. (C) High speed video experimental setup. (D) Simulation of the robot using MBDyn

(E) Leg tip trajectories in simulation. (F) Simulation of the robot running on a bed of $3 \mathrm{~mm}$ particles. 


\section{3) Locomotion experiments}

We ran the DynaRoACH robot on a $75 \mathrm{~cm}$ long, $30 \mathrm{~cm}$ wide trackway filled to a depth of $6 \mathrm{~cm}$ (Fig. 1C). We pressed the particles using a flat plate before each trial to prepare the particles to a closely packed state. Running kinematics were captured by two high speed video cameras (AOS X-PRI) from both top and side views at a frame rate of $200 \mathrm{fps}$. One high contrast dorsal marker was bonded above the robot center of mass to obtain average forward speed; two lateral markers were bonded on the front and rear of the robot body to obtain CoM height (approximated by the average vertical position of the two markers). Stride frequency was determined from the videos.

\section{4) Leg trajectories}

To capture prescribed leg trajectories, the robot was suspended in the air and the leg trajectories of the tip were recorded as the motor rotated. During a cycle, each leg rotated backward about the hip (retraction), lifted-up sideways, and swung forward (protraction) (Fig. 1B). Leg kinematic parameters such as the fore-aft swing angle and lateral lifting angle were determined by tracking the markers on the legs and used to guide tuning of leg trajectories in simulation. We define a stride period $\mathrm{T}$ as the time between the start of two consecutive retraction phases, and stance as when the leg generates ground reaction force (determined from simulation).

\section{B. Simulation}

\section{1) Discrete element method to model contact forces}

To investigate locomotion of DynaRoACH in more detail, a simulation of the robot was developed and coupled to a granular media simulation. This granular simulation used the discrete element method (DEM) to compute the particleparticle and particle-intruder interaction for the $3 \mathrm{~mm}$ diameter glass particles. As in previous work [17], the DEM simulation was validated by matching the forces on intruders moving in the granular medium (e.g., a rod dragged horizontally) with experimental measurements.

In the DEM simulation, the normal contact force between an interacting pair of particles is given by a standard force law [22], a Hertzian repulsion and a velocity dependent dissipation (to account for a coefficient of restitution):

$$
F_{n}=k_{n} \delta^{3 / 2}-G_{n} v_{n} \delta^{1 / 2} ;
$$

the tangential contact force is modeled as Coulomb friction:

$$
F_{s}=\mu F_{n}
$$

where $\delta$ is the deformation (virtual overlap) between contacting particle pairs or particle-intruder pairs, $v_{n}$ the normal component of relative velocity, $k_{n}=2 \times 10^{6} \mathrm{~kg} \mathrm{~s}^{-2} \mathrm{~m}^{-1}$ and $G_{n}=15 \mathrm{~kg} \mathrm{~s}^{-1} \mathrm{~m}^{-1 / 2}$ the contact stiffness and viscoelasticity dissipation coefficient, and $\mu_{[\mathrm{pp}, \mathrm{pi}\}}=\{0.1,0.3\}$ the particleparticle and particle-intruder friction coefficients. The restitution and friction coefficients were experimentally measured and validated in the rod drag experiment [17]. Once the parameters were set in the DEM simulation, the robot locomotion could be accurately predicted over a wide range of conditions. The simulated granular bed $\left(3 \times 10^{5}\right.$ particles $)$ was 60 PD (particle diameter) in width, 15 PD in depth, and 290 PD in length, and had frictionless boundaries (Fig. 1F). At low frequencies we used a shorter granular bed (90 PD) containing $1 \times 10^{5}$ particles to save computation time.

\section{2) Dynamic simulation of the robot}

To model the robot we used a multi-body dynamic simulator, MBDyn [23], which allows time domain simulation of multi-body mechanical systems from first principle equations. MBDyn features a full 3D simulation with six translation and rotation degree-of-freedoms. This is essential for locomotion on the surface during which pitch, roll, and yaw are often present [15].

In the dynamic simulation, the robot was constructed with similar body and leg geometries as the actual robot (Fig. 1D). The modeled robot was composed of 13 individual rigid parts: one box-shaped body, six c-shaped legs, and six linking plates between legs and body. The legs of the actual robot were not perfectly rigid but experimental observations showed little leg deformation during locomotion on granular media. The joints between the link plates and c-legs allowed front-back swing of the legs while the plate-body joints allowed sideway lifting of the legs. Tuning kinematic parameters for the joint movements produced leg trajectories that resembled experimental measurements (Fig. 1E) without mimicking the internal linkage of real robot.

\section{3) Integration of DEM with dynamic simulation}

We combined MBDyn with the DEM code to simulate robot locomotion on granular media via a communication interface (a UNIX socket using $\mathrm{C}++$ ). At each time step, MBDyn integrated the equations of motion for the robot combined with the force and torques calculated from the DEM code. The updated kinematics including position, orientation, velocity, and angular velocity of each part of the robot were then passed back to the DEM code to compute the force and torque on all interacting elements at the next time step. The time step was $1 \mu \mathrm{s}$ set by the particle collision time in DEM.

In addition to the kinematics during locomotion (e.g., CoM position and velocity, stride length, limb penetration depth), the dynamics during locomotion (e.g., net ground reaction force on each limb and tripod) were also determined from the simulation.

\section{Kinematic predictions based on previous work}

Because the small DynaRoACH robot has similarly shaped c-legs to the larger SandBot, we use the rotary walking model developed for SandBot [9] to make two kinematic predictions for the locomotion of the DynaRoACH robot on granular media. We will test these predictions in both experiment and simulation.

First, we predict that both the body height and forward speed will increase during stance and decrease between stances. For walking at low frequencies, leg intrusion speeds will be small enough that granular forces will be dominated by friction, and therefore independent of speed, and increase with depth (hydrostatic-like) [24], [25]. As a result, during each step, the legs should initially slowly penetrate into granular 
medium while the body rests on the surface. As the legs penetrate deeply enough for the lift on the legs to balance the weight and vertical inertial force of the body, the legs will stop penetrating and rotate atop solidified granular media, lifting the body and kinematically propelling it forward. As the legs withdraw from the granular medium, the body will fall and forward speed will decrease to zero. We refer to this as rotary walking in SandBot, and expect to see these features in the small robot.

Second, based on the rotary walking model, we predict that stride length should decrease with stride frequency. In the quasi-static rotary walking mode, stride length is inversely related to leg penetration depth by geometry. As stride frequency increases, because the inertial force of the body during body lift-up increases, the legs must penetrate more deeply, and therefore the stride length will decrease. In addition, the transition from walking to swimming will be triggered by the reduction in stride length - at high enough stride frequency, stride length should become small enough that the legs will encounter previously disturbed material during each step.

\section{RESULTS AND DISCUSSION}

\section{A. Kinematics}

The time-averaged forward speed of the robot measured

\section{Low frequency}
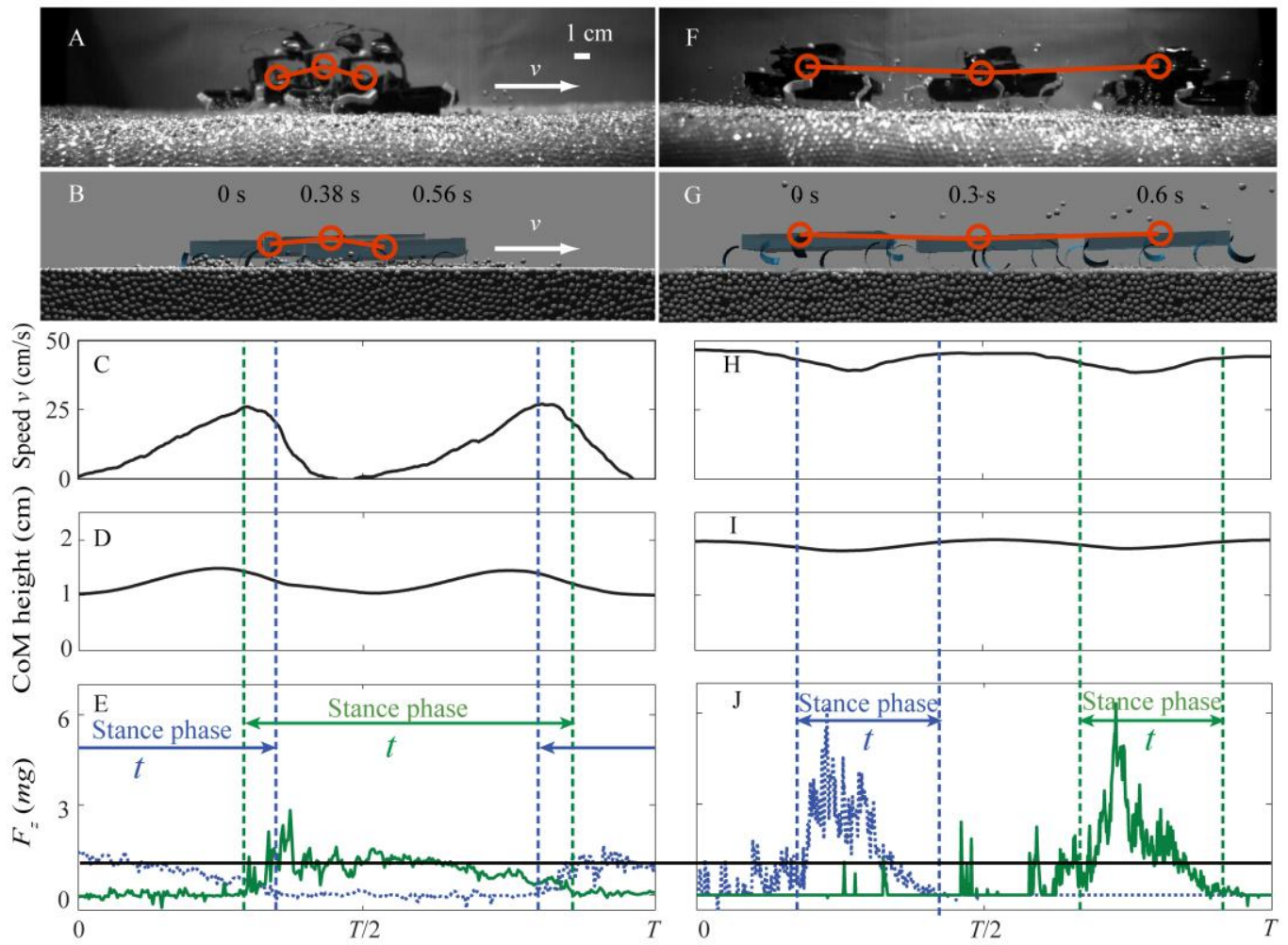

Figure 2. Two locomotion modes observed for DynaRoACH moving on granular media. (A-E) Walking at low frequencies (e.g., 3 Hz). (F-J) Running at high

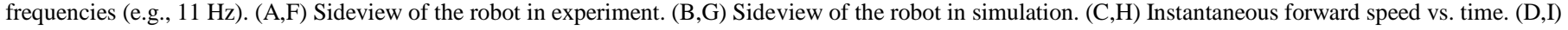
Body height vs. time. (E,J) Vertical ground reaction force on a tripod vs. time. Dashed blue curve and solid green curve are for the two alternating tripods. Black horizontal line indicates body weight $(m g)$. In $(\mathrm{A}, \mathrm{B})$ and $(\mathrm{F}, \mathrm{G})$ the three time instants shown are the start, middle, and end of three different stances. In $(\mathrm{C}$-E) and (H-J) data are shown for simulation only. Duty factor is stance duration $t$ divided by stride period $T$. 
The observed decrease in stride length with stride frequency, increase in body height and forward speed during most of stance, and decrease in body height and forward speed between stances, were in accord with the rotary walking model [9] (Fig. 3A,B, red curve). This suggests that like SandBot, the small DynaRoACH robot also rotarywalked on solidified granular media at low frequencies.

The decrease in body height and forward speed occurred at the start of stance; however, this does not contradict the rotary walking model, but is a consequence of the different leg trajectories of the small robot and SandBot. Because SandBot rotates its legs in circular trajectories, its body must rest on the surface between two tripods, resulting in stance phase that begins during the retraction phases of legs. The small DynaRoACH robot instead uses protraction-retraction leg trajectories, which result in stance phases that begin during protraction phases of the legs.

\section{2) Running at high stride frequencies}

At high stride frequencies (Fig. 2F-J, $11 \mathrm{~Hz}$ ) the DynaRoACH robot exhibited a different locomotor mode than predicted by the rotary walking model. The forward speed of DynaRoACH increased super-linearly with stride frequency (i.e., stride length increased; Fig. 3B). Instantaneous forward speed was always greater than zero and decreased during the first half of stance, and then increased during the second half of stance (Fig. 2H). The average body height was $1.9 \mathrm{~cm}$ above the surface (Fig. $2 \mathrm{~F}, \mathrm{G}, \mathrm{I})$, which was $0.7 \mathrm{~cm}$ ( $58 \%$ of the standing body height $1.2 \mathrm{~cm}$ ) higher than that at low frequencies. Body height decreased by approximately $0.28 \mathrm{~cm}$ (23\% of the standing body height $1.2 \mathrm{~cm}$ ) during the first half of stance and increased by the same amount during the second half of stance. Simulation revealed that grains around the intruding legs remained fluidized throughout the stance phase.

While these kinematics were different from those predicted by the rotary walking model (Fig. 3A,B, red curve), they also differed from the slow surface swimming which SandBot used at high stride frequencies [9], in which the body height remained constant while the belly lay on the surface and forward speed was small $(\sim 1 \mathrm{~cm} / \mathrm{s})$. The decrease in body height and instantaneous forward speed during the first half of stance, the monotonic increase of average forward speed with stride frequency, and the aerial phases observed in the small robot at high stride frequencies resembled those observed in the zebra-tailed lizard running on granular substrates [5], which follows a spring-loaded inverted pendulum (SLIP) model [26]. This suggested that unlike SandBot but like the zebra-tailed lizard, the small robot used a SLIP-like running mode at high frequencies.

\section{B. Vertical ground reaction force}

To understand the mechanism of the transition in locomotor mode from walking at low frequencies to running (but not swimming) at high frequencies, we examined in simulation the vertical ground reaction force, $F_{z}$, on a tripod of legs. For animals and legged robots moving on deformable or yielding substrates such as the surface of water [27] or granular surfaces [5], [9], it is critical to generate sufficient $F_{z}$ to balance the weight and inertial force of the body before legs sink too deeply into the substrate. Averaged over a cycle, lift must equal the body weight, i.e., $\frac{1}{D T} \int F_{z} d t=m g$, where $m g$ is the body weight of the robot, $T$ the cycle period, and $D$ the duty factor defined as the stance duration divided by $\mathrm{T}$.

\section{Low frequency walking High frequency running} on yielding ground on fluidizing ground
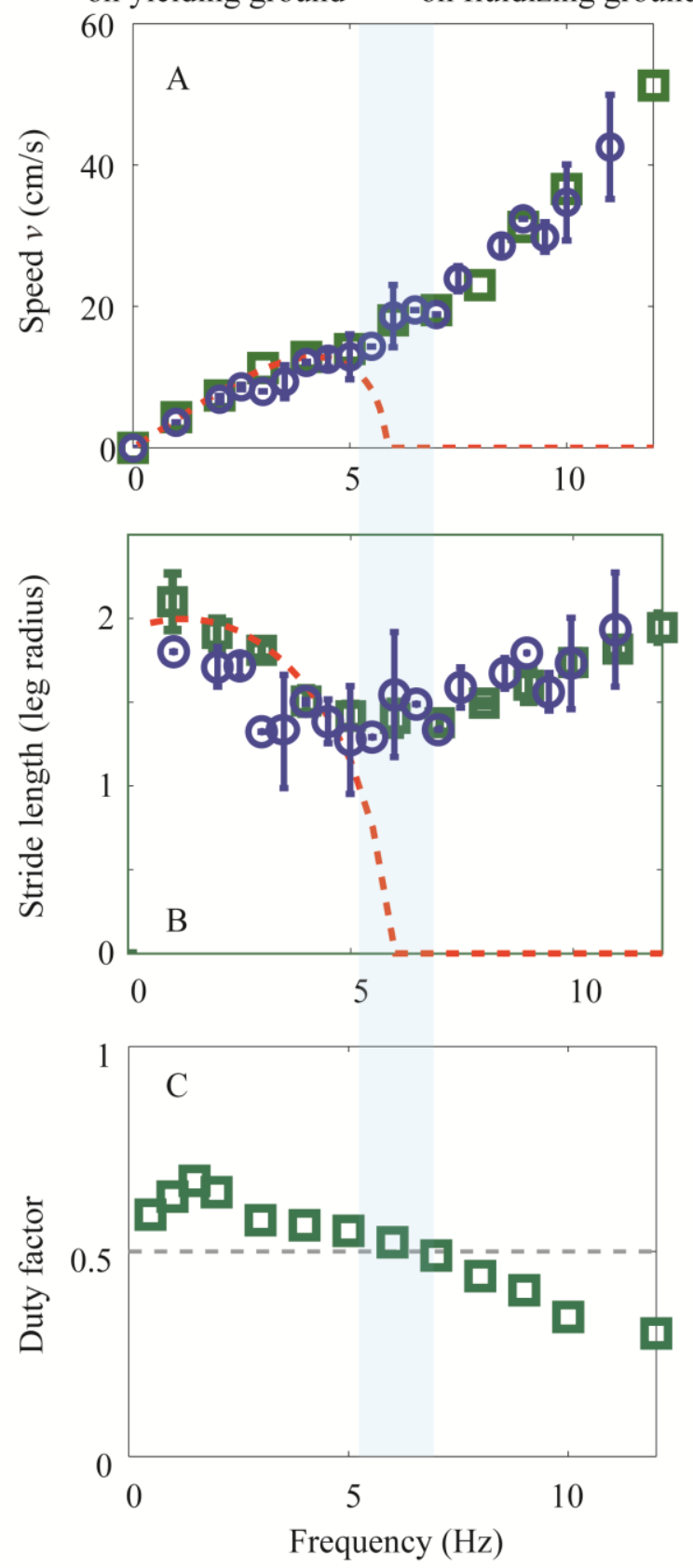

Figure 3. Performance and gait parameters. (A) Average forward speed vs. stride frequency (blue circles: experiment; green squares: simulation) (B) stride length vs. stride frequency (blue circles: experiment; green squares: simulation) (C) duty factor vs. stride frequency (simulation only). Dashed red curves in (A,B) are predictions from the rotary walking model [9]. Error bars indicate standard deviation. 
At low stride frequencies (e.g., $3 \mathrm{~Hz}$ ), because duty factor was greater than (but close to) 0.5 , the $F_{z}$ on both tripods was close to the body weight for most of the cycle (Fig. 2E). As duty factor decreased below 0.5 with increasing stride frequency, the $F_{z}$ on both tripods no longer overlapped, and the magnitude of $F_{z}$ on each tripod increased. The peak of $F_{z}$ increased from $\sim 1 \mathrm{mg}$ at $3 \mathrm{~Hz}$ (Fig. 2E) to $\sim 6-7 \mathrm{mg}$ at $12 \mathrm{~Hz}$ (Fig. 2J). Peak torque on a tripod about the hips measured at $12 \mathrm{~Hz}$ was $10 \mathrm{mN}$-m; this was less than the stall torque of the motor-gearbox system.

The large increase in $F_{z}$ at high frequencies was likely a result of a material response to leg intrusion which differed from the friction-dominated yielding observed in the SandBot study. This is because the SandBot walking model based on the friction-dominated hydrostatic-like forces could only explain the mechanism governing the increase in $F_{z}$ at low frequencies. At low frequencies $(<3 \mathrm{~Hz})$, granular force was friction-dominated, and therefore was assumed to be independent of intrusion speed and to increase with penetration depth. As stride frequency increased, this depth dependent granular force increased due to the increasing inertial force associated with lifting the body [9], resulting in an increasing penetration depth (Fig. 4A). At high stride frequencies $(>3 \mathrm{~Hz}$ ), however, the measured leg penetration depth decreased instead (Fig. 4A), counter to the rotary walking model prediction. In this case, the walking model predicted a decrease in the lift force on the legs, contrary to observations (Fig. 2E, J).

This discrepancy suggests that there must be additional contribution to the force at high frequencies. Examination of leg kinematics in simulation revealed that the vertical penetration speed of the legs increased with stride frequency and reached nearly $1 \mathrm{~m} / \mathrm{s}$ at $12 \mathrm{~Hz}$ (Fig. 4B). It is known that the granular forces during high speed impact are hydrodynamic-like and increase quadratically with impact speed [28], [29]. Our data of lift vs. vertical leg penetration speed (Fig. 5, green squares) fit well to a quadratic with a non-zero $y$-intercept (Fig. 5, red curve), due to the finite yield stress of the medium. We hypothesize that as the vertical leg penetration speeds increased, the inertial force of the grains being accelerated by the legs becomes important and contributes significantly to the vertical ground reaction force. In other words, at high frequencies, instead of swimming, the robot runs on the granular material that behaves like an "inertial fluid", much like the basilisk lizard (Basiliscus), the so-called "Jesus Christ lizard" that runs on the surface of water [27].

We used previous intrusion studies in granular media to estimate the transition frequency for the DynaRoACH robot. Studies of horizontal drag and vertical impact in granular media [24], [28], [29] suggest that inertial effects become important for intrusion speeds beyond $v_{c} \sim(2 g d)^{1 / 2}$, where $d$ is the particle diameter and $g$ the gravitational acceleration. For $3 \mathrm{~mm}$ glass particles, $v_{c}=25 \mathrm{~cm} / \mathrm{s}$. This indicates that hydrodynamic-like force should become significant as the vertical leg penetration speed increases beyond $\sim 25 \mathrm{~cm} / \mathrm{s}$, or as stride frequency increases past $\sim 6 \mathrm{~Hz}$ (Fig. 4B). This matches the observed transition in locomotor mode around 6 $\mathrm{Hz}$ (Fig. 3). We posit that this transition of the propulsion mechanism from low frequency (i.e., low vertical penetration speed) walking on yielding ground to high frequency (i.e., high vertical penetration speed) running on fluidizing ground is generic to locomotion on granular media. However the frequency at which the walk-to-run transition occurs should depend on parameters associated with the granular media as well as the robot morphology and kinematics.

The capability of the small robot to run rapidly at high frequencies on granular media by using hydrodynamic-like forces, in contrast to SandBot's slow swimming, suggests that lightweight locomotors have an advantage when moving on granular surfaces. Indeed, the small robot's legs are relatively large $\left(\sim 1.4 \mathrm{~cm}^{2}\right)$ compared to its body weight $(25$ g) (each leg applies a pressure of $800 \mathrm{~Pa}$ when standing), and can generate enough hydrodynamic-like lift by paddling its legs rapidly to maintain the body well above the surface. By contrast, SandBot's legs are relatively small $\left(\sim 5 \mathrm{~cm}^{2}\right)$ compared to its body weight $(2300 \mathrm{~g})$ (each leg applies a pressure of $\sim 1.5 \times 10^{4} \mathrm{~Pa}$ when standing), and cannot generate enough hydrodynamic-like lift to support the body before the legs sink deeply enough to encounter previously disturbed material over steps and trigger swimming. This may explain why the zebra-tailed lizard, the highestperforming among desert lizards of similar size, has the largest hind feet [5].

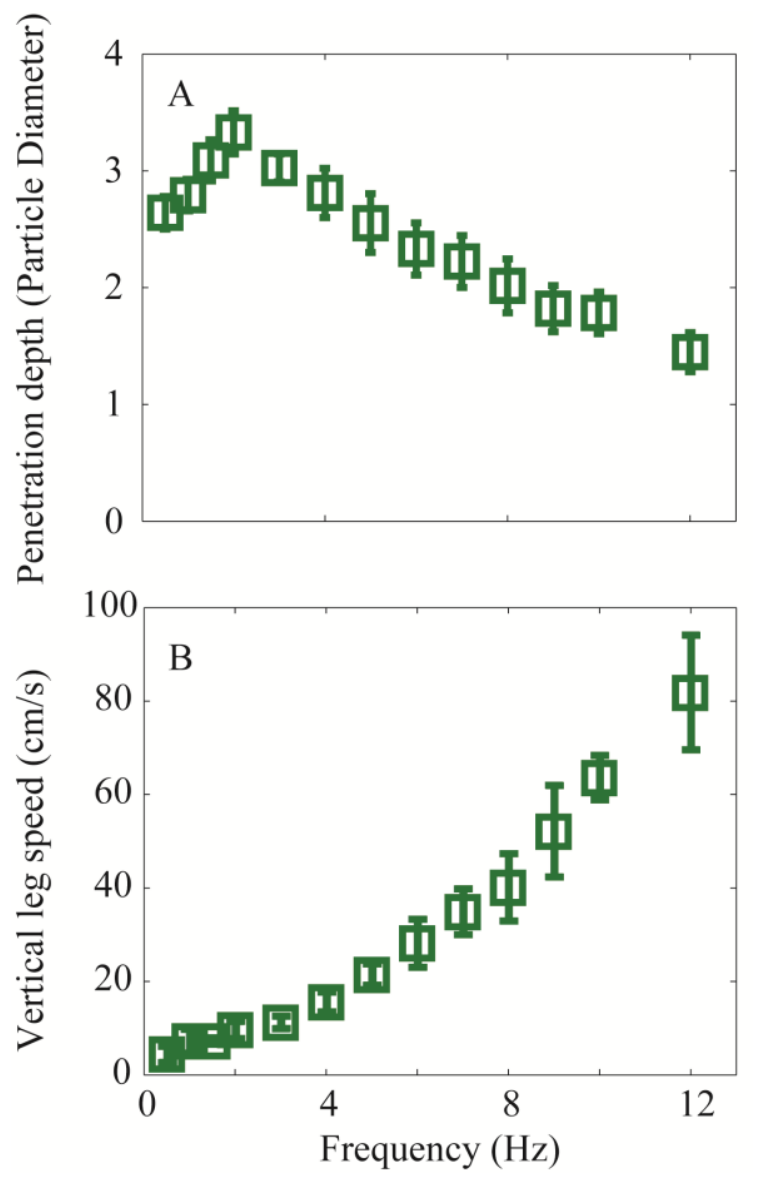

Figure 4. Limb penetration and intrusion speed. (A) Maximal leg penetration depth (measured in particle diameters) vs. stride frequency. (B) Maximum leg vertical penetration speed vs. stride frequency. Error bars indicate standard deviation 


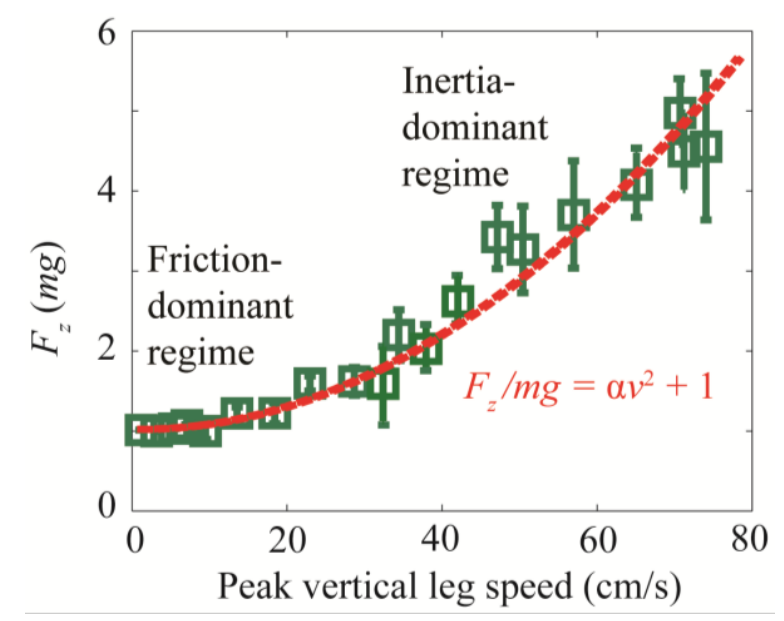

Figure 5. Vertical ground reaction force vs. peak leg penetration speed. Dashed red curve indicates quadratic fit with fitting parameter $\alpha=5.7 \times 10^{-3}$ $\mathrm{cm}^{-2} \mathrm{~s}^{2}$ and with non-zero intercept at $m g$. Error bars indicate standard deviation.

\section{Parameter variation and broader applicability}

Since both the DEM simulation of the granular medium and the MBDyn multi-body analysis are based on first principles (i.e., Newton-Euler equations of motion) and validated empirical models (i.e., Hertzian contact theory, Coulomb model of friction), our results should be applicable to a broad class of granular substrates. The simulation tool therefore allows rapid systematic variation of the properties of the locomotor (e.g., leg geometry, leg trajectories) as well as the granular substrate (e.g., size, density, friction, and hardness of particles). For example, while in experiment it is difficult to alter only one property while not changing others, we can test in simulation the effects of each substrate property. This can provide insight for both design of future multi-terrain robots, and allows testing of biological hypotheses.

To demonstrate the capabilities of our simulation, we analyzed the effect of the coefficients of particle-particle friction, $\mu_{\mathrm{pp}}$. Dramatic changes in locomotor performance were observed when $\mu_{\mathrm{pp}}$ was varied (Fig. 6). For walking at low frequencies (e.g., $3 \mathrm{~Hz}$, Fig. 6, filled green squares), the robot speed increased monotonically with $\mu_{\mathrm{pp}}$, and saturated at high $\mu_{\mathrm{pp}}$. In this regime, forces were dominated by friction. As $\mu_{\mathrm{pp}}$ increased, the legs needed to penetrate less to balance body weight and inertial force. As a result, stride length and speed increased with $\mu_{\mathrm{pp}}$. The saturation of speed at high $\mu_{\mathrm{pp}}$ was a consequence of leg penetration depth approaching zero with increasing $\mu_{\mathrm{pp}}$. Thus stride length could not increase further.

For running at high frequencies (e.g., $12 \mathrm{~Hz}$, Fig. 6, empty green squares), the robot speed increased with $\mu_{\mathrm{pp}}$ for $\mu_{\mathrm{pp}}<0.2$ and decreased with $\mu_{\mathrm{pp}}$ for $\mu_{\mathrm{pp}}>0.2$. The increase of speed with $\mu_{\mathrm{pp}}$ for $\mu_{\mathrm{pp}}<0.2$ is likely because the inertial force provided by the particles being accelerated by the legs increased with $\mu_{\mathrm{pp}}$. We observed that multiple layers of particles directly under and around the legs were fluidized during leg penetration. We hypothesize that the size of the region of accelerating particles increased with $\mu_{\mathrm{pp}}$ because larger $\mu_{\mathrm{pp}}$ facilitated interlocking of the particles.

The decrease of speed with $\mu_{\mathrm{pp}}$ between $0.2<\mu_{\mathrm{pp}}<0.4$ for running at high frequencies is likely a result of reduction in propulsion due to an asymmetric gait. We observed that in this regime, the particles became more tightly interlocked and lost fluidity. The lift force on one tripod alone became sufficient to accelerate the robot up such that the body travelled in the air during the remainder of the cycle such that the other tripod never touched the ground.

As $\mu_{\mathrm{pp}}$ increased above 0.4 , the particles became so tightly interlocked that the granular substrate behaved like rigid ground. Locomotion became unstable as the robot started bouncing erratically, and movement direction changed randomly. This is similar to the unstable motion of the robot on rigid ground [15]. Forward speed was not well defined in this regime. The perfectly rigid legs of the robot used in simulation may have contributed to this instability by generating force spikes during substrate impact [30].

\section{CONCLUSIONS}

Inspired by the high performing desert animals moving on granular media, we studied the locomotion of a lightweight, bio-inspired, legged robot on a granular substrate and developed an experimentally validated computer simulation of the locomotion. Kinematics measured in simulation matched experiment and enabled examination of ground reaction forces responsible for the high locomotor performance. The small robot displayed a transition in locomotor mode from walking at low frequencies to running at high frequencies. At low frequencies, hydrostatic-like forces generated during the yielding of the granular material ultimately led to solidification of the material, and the robot moved as if it were walking on a solid surface. At high frequencies, however, the inertia of the grains being accelerated became important and forces became hydrodynamic-like. In this regime the robot ran rapidly by paddling the legs on fluidized granular material which behaved like an "inertial fluid".

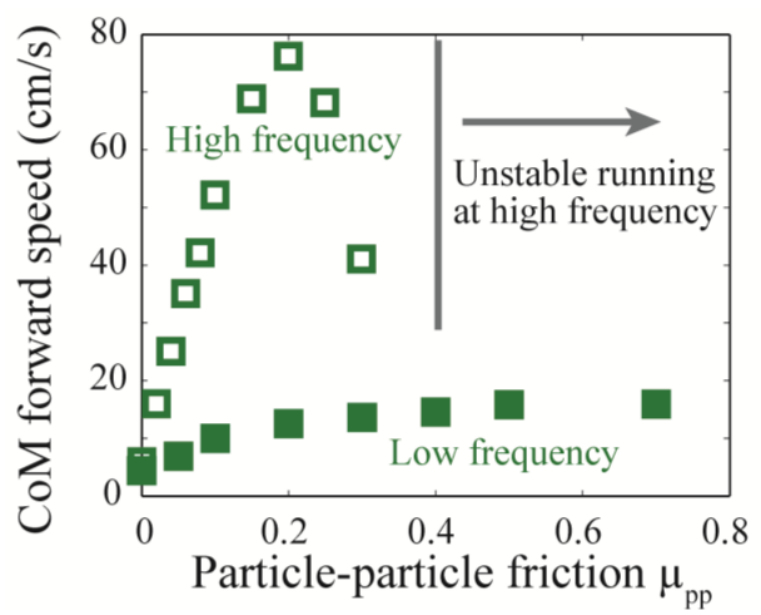

Figure 6. Robot average speed upon variation of particle-particle friction in simulation (filled green squares: $3 \mathrm{~Hz}$, empty green squaress: $12 \mathrm{~Hz}$ ). 
Our results revealed that lightweight robots can achieve high locomotor performance on granular media by exploiting fluid properties of the granular material. This locomotion mode is distinct from previously observed low frequency yielding walking strategy, and provides a better understanding of fundamental locomotive modes for a broad class of granular substrates. The lightweight robot platform enables detailed examination of legged locomotion, and provides likely the best model to date of a robot running on granular media. In addition, the integrated simulation tool we developed can be used to systematically test the effect of both locomotor and substrate properties on locomotor performance, which could guide the design, control and power consumption estimates for high-performing multiterrain robot platforms.

Finally, we note that while experiment and simulation allow detailed investigation of mechanics of movement on granular media, a complementary approach is needed, that of low order dynamical models that can be used to gain insight into the critical mechanics of dynamical running. In our future work, we will investigate if a dynamic force law that describes the hydrodynamic-like forces during high speed leg intrusions can be obtained from measurements in DEM simulation. We posit that a generalized locomotion model similar to the Spring-Loaded Inverted Pendulum (SLIP) [26] can be developed based on the force law, and can extend our current study to more generalized conditions. This generalized model will shed light on the locomotor dynamics of legged animals and robots on granular media, as well as guide development for analytically tractable low order models.

\section{ACKNOWLEDGMENTS}

We thank Jeff Shen for the help with data collection, and Yang Ding and Paul Umbanhowar for helpful discussion.

\section{REFERENCES}

[1] U. Saranli, M. Buehler, and D. E. Koditschek, "RHex: A Simple and Highly Mobile Hexapod Robot," The International Journal of Robotics Research, vol. 20, no. 7, pp. 616-631, 2001.

[2] R. T. Schroer, M. J. Boggess, R. J. Bachmann, R. D. Quinn, and R. E. Ritzmann, "Comparing cockroach and Whegs robot body motions," in Proceedings of the 2004 IEEE International Conference on Robotics and Automation, 2004, vol. 4, pp. 3288-3293.

[3] C. Wright et al., "Design of a modular snake robot," Proceedings of the 2007 IEEE/RSJ International Conference on Intelligent Robots and Systems, pp. 2609-2614, Oct. 2007.

[4] S. Kim, M. Spenko, and S. Trujillo, "Whole body adhesion: hierarchical, directional and distributed control of adhesive forces for a climbing robot," Robotics and Automation, no. April, pp. 10-14, 2007.

[5] C. Li, S. T. Hsieh, and D. I. Goldman, "The effect of substrate on the running mechanics and energetics of the zebra-tailed lizard (Callisaurus draconoides)," Journal of Experimental Biology, in press.

[6] J. Matson, "Unfree Spirit: NASA's Mars Rover Appears Stuck for Good," Scientific American, vol. 302, no. 4, p. 16, 2010.

[7] H. M. Jaeger, S. R. Nagel, and R. P. Behringer, "The physics of granular materials," Physics Today, vol. 49, p. 32, 1996.

[8] R. M. Nedderman, Statics and Kinematics of Granular Materials. Cambridge: Cambridge University Press, 1992.
[9] C. Li, P. B. Umbanhowar, H. Komsuoglu, D. E. Koditschek, and D. I. Goldman, "Sensitive dependence of the motion of a legged robot on granular media," Proceedings of the National Academy of Sciences of the United States of America, vol. 106, no. 9, pp. 3029-3034, 2009.

[10] R. J. Wood, R. Sahai, E. Steltz, and R. S. Fearing, "Microrobot design using fiber reinforced composites," Journal of Mechanical Design, vol. 130, no. May, pp. 1-11, 2008.

[11] A. M. Hoover, S. Burden, X. Fu, S. S. Sastry, and R. S. Fearing, "Bio-inspired design and dynamic maneuverability of a minimally actuated six-legged robot," Biomedical Robotics and Biomechatronics (BioRob), pp. 869-876, 2010.

[12] P. Birkmeyer, K. Peterson, and R. S. Fearing, "DASH: A dynamic 16g hexapedal robot," in Intelligent Robots and Systems, 2009. IROS 2009. IEEE/RSJ International Conference on, 2009, pp. 2683-2689.

[13] W. Mosauer, "Adaptive convergence in the sand reptiles of the Sahara and of California: A study in structure and behavior," Copeia, vol. 1932, no. 2, pp. 72-78, Jul. 1932.

[14] C. S. Crawford, Biology of Desert Invertebrates. New York: Springer, 1981.

[15] C. Li, A. M. Hoover, P. Birkmeyer, P. B. Umbanhowar, R. S. Fearing, and D. I. Goldman, "Systematic study of the performance of small robots on controlled laboratory substrates," Proceedings of SPIE Defense, Security, and Sensing Conference, 7679:76790Z (1-13), 2010.

[16] S. Vogel, Life in moving fluids: the physical biology of flow. Princeton Univ Pr, 1996.

[17] R. D. Maladen, Y. Ding, P. B. Umbanhowar, A. Kamor, and D. I. Goldman, "Mechanical models of sandfish locomotion reveal principles of high performance subsurface sand-swimming," Journal of The Royal Society Interface, vol. 8, no. 62, pp. 1332-1345, Mar. 2011.

[18] A. A. Biewener and R. J. Full, "Force platform and kinematic analysis," in Biomechanics: Structures and Systems: A Practical Approach, Oxford University Press, 1992, pp. 45-73.

[19] W. W. Dickinson and J. D. Ward, "Low depositional porosity in eolian sands and sandstones, Namib Desert," Journal of Sedimentary Research, vol. 64, no. 2a, pp. 226-232, 1994.

[20] M. Jerkins, M. Schr, T. J. Senden, M. Saadatfar, and T. Aste, "Onset of mechanical stability in random packings of frictional spheres," Physical Review Letters. vol. 101, no. 1, pp. 1-4, 2008.

[21] N. Gravish, P. B. Umbanhowar, and D. I. Goldman, "Force and flow transition in plowed granular media," Physical Review Letters, vol. 105, no. 12, pp. 208301(1-4), Sep. 2010.

[22] J. Shäfer, S. Dippel, and D. Wolf, "Force schemes in simulations of granular materials," Journal de physique I, vol. 6, no. 1, pp. 5-20, 1996.

[23] G. L. Ghiringhelli, P. Masarati, and P. Mantegazza, "MultiBody Analysis of a Tiltrotor Con guration Multi-Body Formulation," Nonlinear Dynamics, vol. 19, no. 4, pp. 333-357, 1999.

[24] R. Albert, M. A. Pfeifer, A. Barabási, and P. Schiffer, "Slow Drag in a Granular Medium," Physical Review Letters, pp. 205-208, 1999.

[25] G. Hill, S. Yeung, and S. a Koehler, "Scaling vertical drag forces in granular media," Europhysics Letters (EPL), vol. 72, no. 1, pp. 137-143, Oct. 2005.

[26] R. Blickhan, "The spring-mass model for running and hopping," Journal of Biomechanics, vol. 22, no. 11-12, pp. 1217-1227, 1989.

[27] J. W. Glasheen and T. A. Mcmahon, "A hydrodynamic model of locomotion in the Basilisk Lizard," Nature, vol. 380, no. 6572, pp. 340-341, 1996.

[28] H. Katsuragi and D. J. Durian, "Unified force law for granular impact cratering," Nature Physics, vol. 3, no. 6, pp. 420-423, Apr. 2007.

[29] D. I. Goldman and P. B. Umbanhowar, "Scaling and dynamics of sphere and disk impact into granular media," Physical Review E, vol. 77, no. 2, pp. 021308(1-14), Feb. 2008.

[30] S. Kim, J. E. Clark, and M. R. Cutkosky, "iSprawl: Design and tuning for high-speed autonomous open-loop running," The International Journal of Robotics Research, vol. 25, no. 9, pp. 903-912, 2006. 